\title{
铜催化芳基(或烷基)卤化物选择性烯丙基化反应研究
}

\author{
韩博士 ${ }^{a}$ 时 郑 ${ }^{a}$ 何慧红*,a 张兴华*,a,b \\ ( ${ }^{a}$ 上海应用技术大学化学与环境工程学院 上海 201418) \\ ${ }^{b}$ 宁波大学新药技术研究院 浙江宁波 315211)
}

\begin{abstract}
摘要 以溴代芳烃和烯丙基乙酸酯为反应原料, 发展了一种铜催化高效构建碳碳键的交叉偶联方法. 该方法通过 “一 锅法” 策略，有效避免了预制金属有机试剂的使用，在较温和条件下即可实现烯丙基类化合物的高区域选择性制备.

关键词 铜催化; 烯丙基化; 区域选择性
\end{abstract}

\section{Study on the Copper-Catalyzed Selective Allylation of Aryl (or Alkyl) Halides}

\author{
Han, Boshi $^{a} \quad$ Shi, Zheng ${ }^{a} \quad$ He, Huihong*,a Zhang, Xinghua ${ }^{*, a, b}$ \\ $\left({ }^{a}\right.$ School of Chemical and Environmental Engineering, Shanghai Institute of Technology, Shanghai 201418) \\ $\left({ }^{b}\right.$ Institute of Drug Discovery Technology, Ningbo University, Ningbo, Zhejiang 315211)
}

\begin{abstract}
A practical method for the $\mathrm{Cu}$-catalyzed coupling of aryl (or alkyl) halides with allylic acetates was described. This procedure effectively avoids the use of preformed Grignard reagent and affords allyl products with high to excellent regioselectivity under relatively mild condition.

Keywords copper-catalyzed; allylation; regioselectivity
\end{abstract}

烯丙基化反应是有机合成中引入 C-3 结构单元最为 简单有效的方法之一，其显著特点是化学和区域选择性 多变, 可根据特定的要求和用途进行调控, 被广泛应用 于生物活性物质以及天然产物的合成 ${ }^{[1]}$. 近年来, 由过 渡金属(钯、铱、镍、铜等)催化的烯丙基化取代反应取 得了较大发展 ${ }^{[2]}$, 其中又以过渡金属催化的 Tsuji-Trost 反应研究最为深入, 大多通过金属有机试剂与烯丙基化 合物的交叉偶联实现含有烯丙基结构单元的碳碳键高 选择性构建 ${ }^{[3]}$. 近年来, $\mathrm{Liu}^{[4]}$, Sun ${ }^{[5]}$, Wangelin ${ }^{[6]}$ 等相继 报道了过渡金属催化下多种卤代芳(烷)基化合物与亲电 试剂的偶联反应，通过金属镁的参与以 “一锅法” 策略 实现了 $\mathrm{C}-\mathrm{C}$ 键的简洁、高效合成, 有效避免了预制金 属有机试剂的使用. 此外, Gong ${ }^{[7]}$, Weix ${ }^{[8]}$, Gosmini ${ }^{[9]}$ 等 采用过渡金属 $\mathrm{Ni}, \mathrm{Co}$ 等催化, 也实现了卤代烃与烯丙基 乙酸酯的烯丙基化偶联, 但产物的区域选择性受底物结 构的影响较大.

相比之下, 通过金属镁活化实现过渡金属催化下卤
代烃与烯丙基乙酸酯的烯丙基化反应少有报道. 2010 年, Wangelin 等 ${ }^{[66]}$ 报道了铁催化下澳代芳烃和烯丙基乙 酸酯的偶联反应，但烯丙基乙酸酯的结构对其区域选择 性影响较大. 基于铜催化剂的自身优势(催化活性较高、 成本较低及催化稳定性较差的亲核试剂参与烯丙基化 反应 $)^{[2 \mathrm{~b}-2 \mathrm{c}]}$, 本文对金属铜盐催化下卤代烃与烯丙基乙 酸酯的交叉偶联反应进行了研究，在有效避免预制格氏 试剂使用的同时，也实现了一系列烯丙基类化合物的高 效、高区域选择性合成.

\section{1 结果与讨论}

\section{1 反应条件优化}

首先, 选取乙酸肉桂酯(1a)和对溴甲苯 (2a) 作为模 型反应底物, $\mathrm{CuSCN}$ 为催化剂, $\mathrm{PPh}_{3}$ 为配体, $\mathrm{LiCl}$ 为添加 剂, 四氢呋喃(THF)为溶剂在金属镁的活化下对反应条 件进行篮选. 在 $30{ }^{\circ} \mathrm{C}$ 下反应 $6 \mathrm{~h}$ 后以 $68 \%$ 的收率获得 产物 3a(表 1, Entry 1), ${ }^{1} \mathrm{H}$ NMR 表明芳基的烯丙基化反

* Corresponding authors. E-mail: xhzhang@sit.edu.cn; eilynn1207@126.com Received August 10, 2020; revised September 17, 2020; published online October 13, 2020. Project supported by the National Natural Science Foundation of China (No. 21871182). 国家自然科学基金(No. 21871182)资助项目. 
应几乎完全生成了线性产物( $\alpha$ 选择), 具有极高的区域 选择性. 进一步对催化剂进行笁选, 发现以 $\mathrm{CuBr}$ 为催 化剂时反应效果最佳，反应收率可以提升至 74\%(表 1, Entries 2 4). 此外, 对反应的配体进行了篮选, 结果表 明, 选用含氮配体(如 1,10-phen, TMEDA)和含磷配体 (DPPM) 时效果不佳, 反应收率均低于 $\mathrm{PPh}_{3}$ (表 1, Entries 5 7); 虽然采用配体 $\mathrm{PCy}_{3}$ 时反应的收率可升高至 74\%, 但是副产物 $4 \mathrm{a}$ (原料 $2 \mathrm{a}$ 自身偶联产物)也相对较高(表 1, Entry 8). 为了进一步提高反应收率, 对锂盐也进行了考 察, 结果显示不加锂盐时反应收率仅有 $66 \%$ (表 1, Entry 9), 而当选用 $\mathrm{LiBr}$ 为添加剂时, 反应效率进一步提高到 78\%(表 1, Entry 10), 这一现象表明锂盐在反应过程中 起到了一定的促进作用. 此外, 以金属锌、锰替代镁后 均不能使反应顺利进行(表 1, Entry 11). 在研究溶剂对 反应的影响时(表 1, Entry 12)发现, 溶剂的选择对反应 有着重要影响, 反应只能在四氢呋喃环境中进行, 而选 取甲苯、二氯甲烷或乙腈为溶剂则无法得到目标产物. 通过对反应温度的考察(表 1 , Entries 13 15)发现, 反应 收率随反应温度的升高而升高. 值得注意的是, 提高反 应温度的同时还可以有效抑制反应副产物 $4 \mathrm{a}$ 的生成.

表 1 反应条件的优化 ${ }^{a}$

Table 1 Optimization of reaction conditions

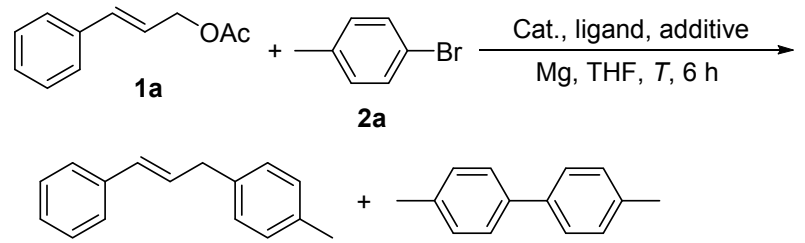

3a

$4 a$

\begin{tabular}{cllccl}
\hline Entry & Cat. & Ligand & Additive & $T /^{\circ} \mathrm{C}$ & $\begin{array}{l}\text { Yield }^{b} \% \\
\text { of 3a/4a }\end{array}$ \\
\hline 1 & $\mathrm{CuSCN}$ & $\mathrm{PPh}_{3}$ & $\mathrm{LiCl}$ & 30 & $68 / 11$ \\
2 & $\mathrm{CuI}$ & $\mathrm{PPh}_{3}$ & $\mathrm{LiCl}$ & 30 & $70 / 11$ \\
3 & $\mathrm{CuCl}$ & $\mathrm{PPh}_{3}$ & $\mathrm{LiCl}$ & 30 & $61 / 12$ \\
4 & $\mathrm{CuBr}$ & $\mathrm{PPh}_{3}$ & $\mathrm{LiCl}$ & 30 & $74 / 10$ \\
5 & $\mathrm{CuBr}$ & $1,10-\mathrm{Ph} n$ & $\mathrm{LiCl}$ & 30 & $66 / 7$ \\
6 & $\mathrm{CuBr}$ & $\mathrm{TMEDA}$ & $\mathrm{LiCl}$ & 30 & N.R. \\
7 & $\mathrm{CuBr}$ & $\mathrm{DPPM}$ & $\mathrm{LiCl}$ & 30 & $59 / 9$ \\
8 & $\mathrm{CuBr}$ & $\mathrm{PCy}_{3}$ & $\mathrm{LiCl}$ & 30 & $74 / 12$ \\
9 & $\mathrm{CuBr}$ & $\mathrm{PPh}_{3}$ & - & 30 & $66 / 12$ \\
10 & $\mathrm{CuBr}$ & $\mathrm{PPh}_{3}$ & $\mathrm{LiBr}$ & 30 & $78 / 14$ \\
$11^{\mathrm{c}}$ & $\mathrm{CuBr}$ & $\mathrm{PPh}_{3}$ & $\mathrm{LiBr}$ & 30 & N.R. \\
$12^{\mathrm{d}}$ & $\mathrm{CuBr}$ & $\mathrm{PPh}_{3}$ & $\mathrm{LiBr}$ & 30 & N.R. \\
13 & $\mathrm{CuBr}$ & $\mathrm{PPh}_{3}$ & $\mathrm{LiBr}$ & 0 & $75 / 16$ \\
14 & $\mathrm{CuBr}$ & $\mathrm{PPh}_{3}$ & $\mathrm{LiBr}$ & 60 & $80 / 6$ \\
$\mathbf{1 5}$ & $\mathrm{CuBr}$ & $\mathbf{P P h}_{\mathbf{3}}$ & $\mathbf{L i B r}$ & $\mathbf{8 0}$ & $\mathbf{8 6} / \mathbf{3}(\mathbf{8 6})^{e}$ \\
\hline
\end{tabular}

${ }^{a}$ Reaction conditions: 1a $(0.5 \mathrm{mmol}), \mathbf{2 a}(1.0 \mathrm{mmol})$, catalyst $(10 \mathrm{~mol} \%), \mathrm{Mg}$ (2.4 equiv.), ligand (20 mol\%), additive (1.0 equiv.), THF $(1.5 \mathrm{~mL}), 6 \mathrm{~h} .{ }^{b}$ Yield determined by ${ }^{1} \mathrm{H}$ NMR using 4-dimethylaminopyridine as an internal standard. ${ }^{c} \mathrm{Zn}$ or Mn instead of Mg. ${ }^{d}$ Toluene, dichloromethane or MeCN instead of THF. ${ }^{e}$ Isolated yield.
因此，最终的反应条件确定为: $\mathrm{CuBr}$ 为催化剂, $\mathrm{PPh}_{3}$ 为 配体, $\mathrm{LiBr}$ 为促进剂, 四氢呋喃为溶剂, 在金属镁的活 化下于 $80{ }^{\circ} \mathrm{C}$ 反应 $6 \mathrm{~h}$ (表 1 , Entry 15).

\section{2 底物普适性研究}

在确定了最优反应条件后，对卤代芳烃底物的适用 范围进行了考察(表 2). 其中对碘甲苯、对溴甲苯参与的

表 2 卤代芳烃拓展

Table 2 Expansion of aryl halides
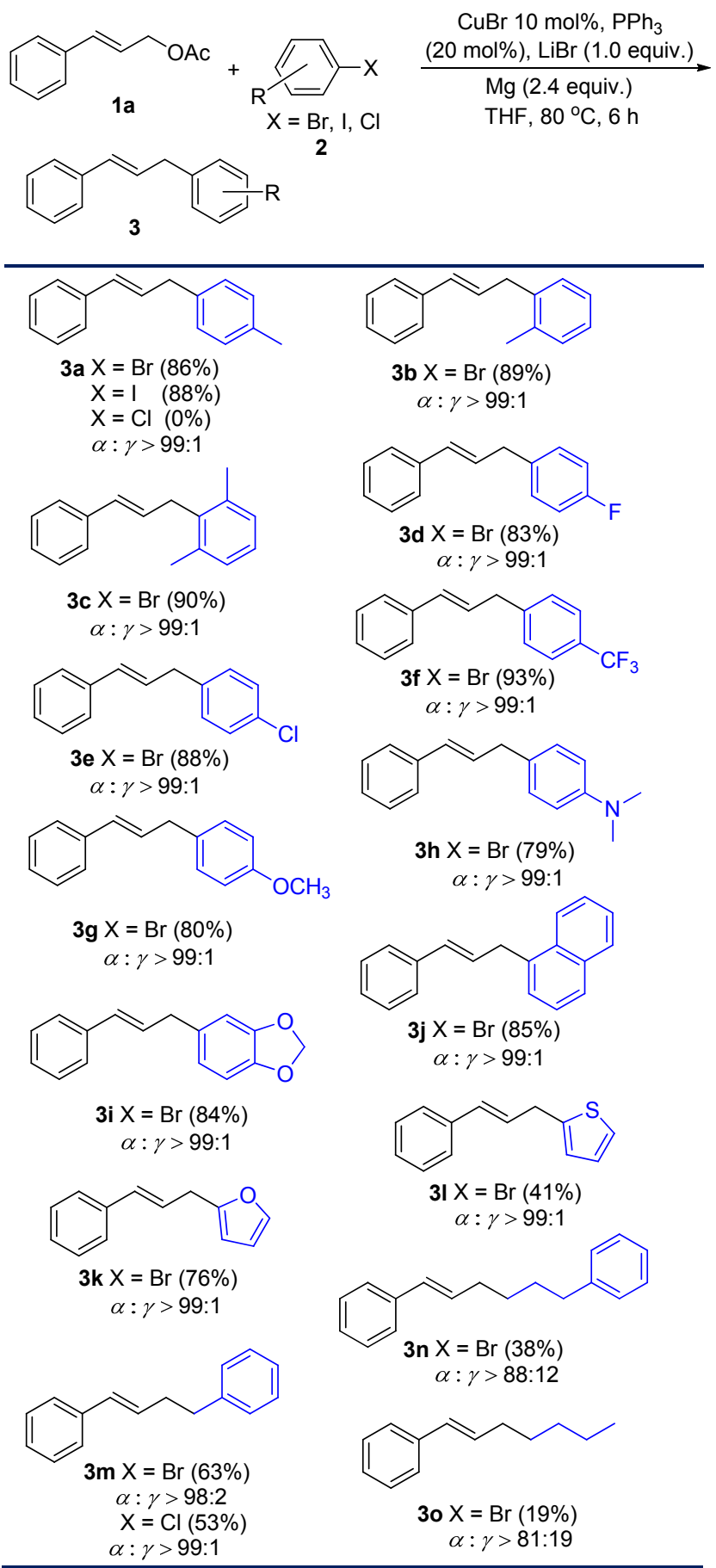

Reaction conditions: 1a (0.5 mmol), 2 (1.0 mmol), $\mathrm{CuBr}(10 \mathrm{~mol} \%), \mathrm{PPh}_{3}(20$ mol\%), $\mathrm{Mg}$ (2.4 equiv.), $\mathrm{LiBr}$ (1.0 equiv.), THF $(1.5 \mathrm{~mL}), 80{ }^{\circ} \mathrm{C}, 6 \mathrm{~h}$. 
反应均能以较高产率生成目标化合物 $\mathbf{3 a}$, 但对氯甲苯 则不适合此类反应. 此外, 改变芳环上取代基的位置对 反应产率的影响较小, 均能较高产率地获得目标化合物 (86\% 90\%，3a 3c). 同样，对于芳环上取代基的电子 效应也进行了考察, 对于溴代芳香化合物, 供电子效应 与给电子效应对还原偶联反应的影响并不是很大, 都能 以较好的收率得到目标化合物. 但总体而言, 芳环上含 吸电子效应基团的底物( $83 \% \sim 93 \%, 3 \mathbf{3 d} \sim \mathbf{3 f}$ )较含给电子 效应基团的底物( $80 \% \sim 84 \%, 3 \mathbf{g} \sim 3 \mathbf{j}$ ) 更利于反应的进 行, 产率偏高. 同时对溴代杂环化合物进行了考察, 结 果表明该反应方法同样适用于含有呋喃、噻吩类杂环化 合物偶联，并以中等产率得到相应的目标化合物 $(76 \%$, 3k; $41 \%, 3 \mathbf{l})$. 此外, 与卤代芳烃相比, 卤代芐烃仍能以 高区域选择性、中等收率得到目标化合物(3m). 但烷基 溴代物对反应的影响较大, 结果表明烷基溴代物的烯丙 基化反应收率及产物的区域选择性较芳基溴代物有较 大差距 (3n, 3o).

对于反应底物烯丙基乙酸酯的适用范围也进行了 考察(表 3), 实验结果表明该反应体系对于一级和二级 烯丙基乙酸酯都具有较好的普适性. 对于一级烯丙基乙 酸酯, 芳环含给电子或吸电子基团都以较高产率及区域 选择性获得目标产物 $(83 \%, \mathbf{3 a a} ; 87 \%, \mathbf{3 a b})$. 研究发现该 方法同样适用于杂环噻吩烯丙基乙酸酯的偶联反应 $(85 \%, 3 \mathrm{ac})$. 此外, 烯丙基 $\beta$ 位的取代基效应对此类反应 的影响较小, 从( $E$ )-2-甲基-3-苯基烯丙基乙酸酯(1e) 出 发也可以实现此类碳碳键的高选择性偶联 $(82 \%, \alpha: \gamma>$ 99: 1, 3ad). 值得注意的是, 产物 3af 和 3ag 较 3ae 具有 更好的产率，同时也获得了较高的区域选择性( $79 \%$, $\alpha: \gamma>99: 1$, 3af; $81 \%, \alpha: \gamma>99: 1$, 3ag). 对于二级烯 丙基乙酸酯, 支链长度及空间位阻对反应的收率及区域 选择性影响较小 $(80 \% \sim 97 \%, \alpha: \gamma \geqslant 97: 3$, 3ah 3al). 芳环上的吸电子基团(如 3an)较供电子基团(如 3am)更 有利于反应产物的获得 $(85 \%, 3 \mathrm{am} ; 91 \%, 3 \mathrm{an})$, 但其区 域选择性有所降低 $(\alpha: \gamma=98: 2, \mathbf{3 a m} ; \alpha: \gamma=99: 5, \mathbf{3 a n})$.

\section{3 反应机理研究}

为了更好地了解反应历程, 开展了如 Scheme 1 所 示的分步控制实验. 通过预制合成格氏试剂, 然后在 $\mathrm{CuBr}$ 催化下与乙酸肉桂酯反应也可以得到预期化合物 3a (Scheme 1a, 51\%), 说明反应历程中经历了生成格氏 试剂的过程. 此外, 为探究锂盐对反应的影响, 在上述 反应条件中加入 $\mathrm{LiBr}$, 也顺利得到了预期产物 $\mathbf{3 a}$ (Scheme 1b,71\%), 虽然较标准反应条件下的产率仍有 一定差距, 但是较预制格氏试剂参与的反应产率有了明 显的提升. 根据文献报道 ${ }^{[10]}, \mathrm{LiBr}$ 在反应中不仅能加速 格氏试剂的生成并对其起到较好的稳定作用, 还可以提
表 3 烯丙基乙酸酯的拓展

Table 3 Expansion of allyl acetates
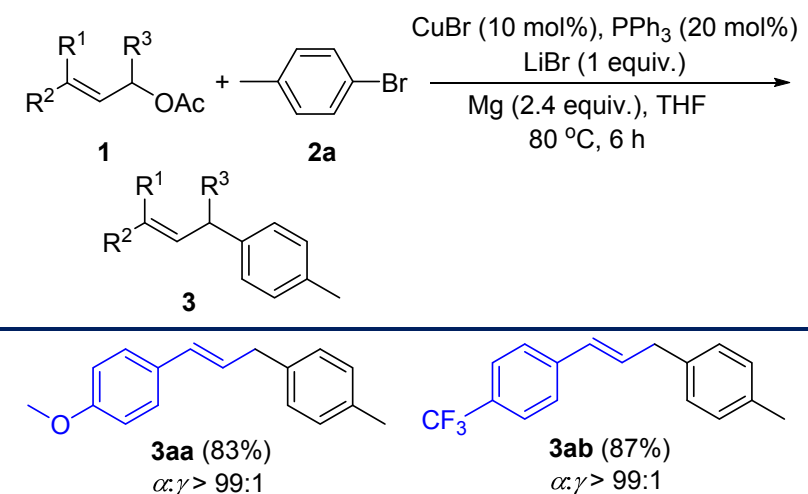

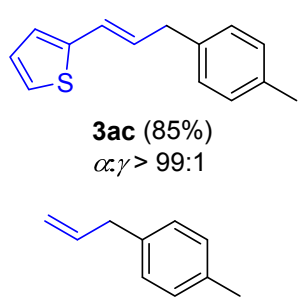

3ae $(56 \%)$

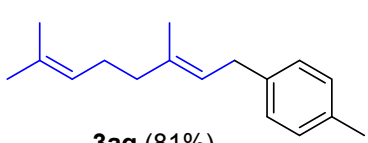

3ag $(81 \%)$

$\alpha \cdot \gamma>99: 1$

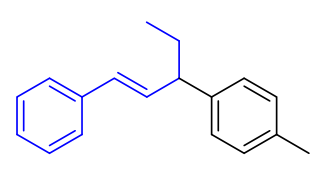

3ai (89\%)

$\alpha \cdot \gamma=99: 1$

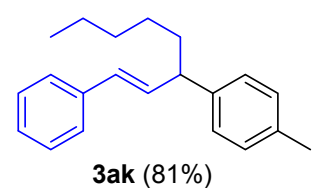

$\alpha: \gamma=97: 3$

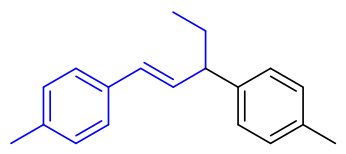

3am (85\%)

$\alpha: \gamma=98: 2$

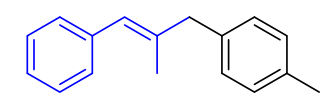

3ad (82\%)

$\alpha: \gamma>99: 1$

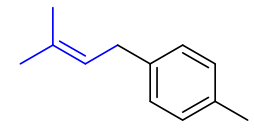

3af $(79 \%)$

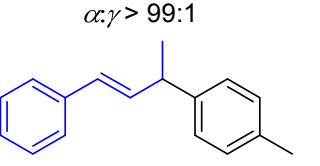

3ah (93\%)

$\alpha \cdot \gamma=97: 3$

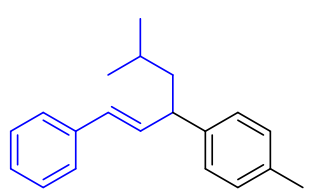

3aj (97\%)

$\alpha: \gamma=98: 2$
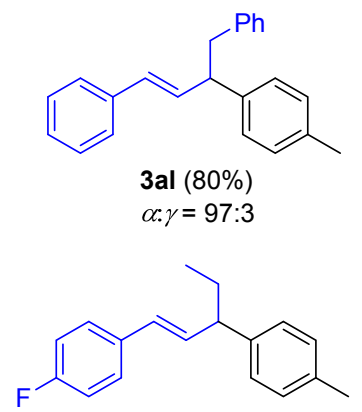

3an (91\%)

$\alpha: \gamma=95: 5$
${ }^{a}$ Reaction conditions: $1(0.5 \mathrm{mmol}), \mathbf{2 a}(1.0 \mathrm{mmol}), \mathrm{CuBr}\left(10 \mathrm{~mol}^{\circ}\right), \mathrm{PPh}_{3}(20$ mol\%), $\mathrm{Mg}$ (2.4 equiv.), $\mathrm{LiBr}\left(1.0\right.$ equiv.), THF (1.5 mL), $80{ }^{\circ} \mathrm{C}, 6 \mathrm{~h}$

高格氏试剂的反应活性，有利于反应的顺利进行。

结合以上实验结果和现有文献报道 ${ }^{[3-9]}$, 提出了以 下可能的反应途径(Scheme 2): $\mathrm{LiBr}$ 促进芳基溴和 $\mathrm{Mg}$ 生

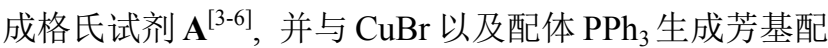
合物 $\mathbf{B}^{[11,3 b]}$; 配合物 $\mathbf{B}$ 进一步和格氏试剂生成二芳基铜 试剂 $\mathbf{C}^{[11-12]}$, 并在锂离子的协同作用下与烯丙基乙酸酯 
(a)
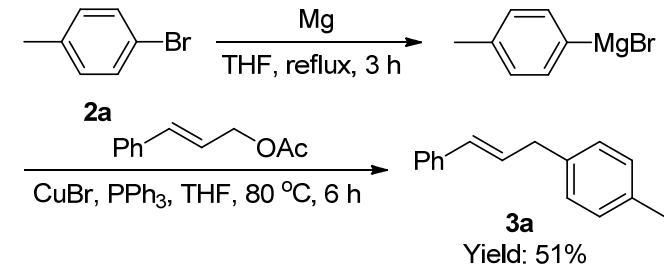

(b)

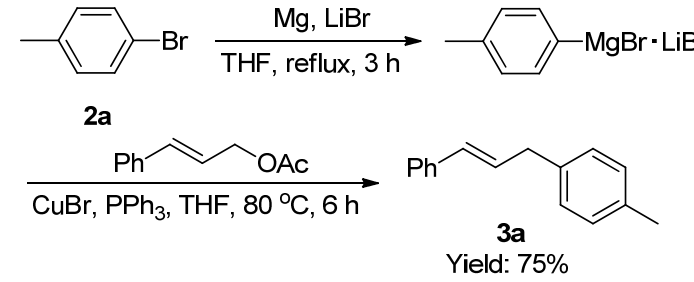

图式 1 控制实验

Scheme 1 Control experiments

不饱和键配位生成 $\pi$-烯丙基 $\mathrm{Cu}(\mathrm{I})$ 金属中间体 $\mathbf{D}^{[13]}$; 随 后经历氧化加成生成 $\mathrm{Cu}(\mathrm{III})$ 中间体 $\mathbf{E}^{[14]}, \mathbf{E}$ 还原消除释 放出 $\mathrm{Cu}(\mathrm{I})$ 配合物 $\mathbf{B}$ 的同时, 高区域选择性( $\alpha$ 选择)的烯 丙基化产物得以构建.

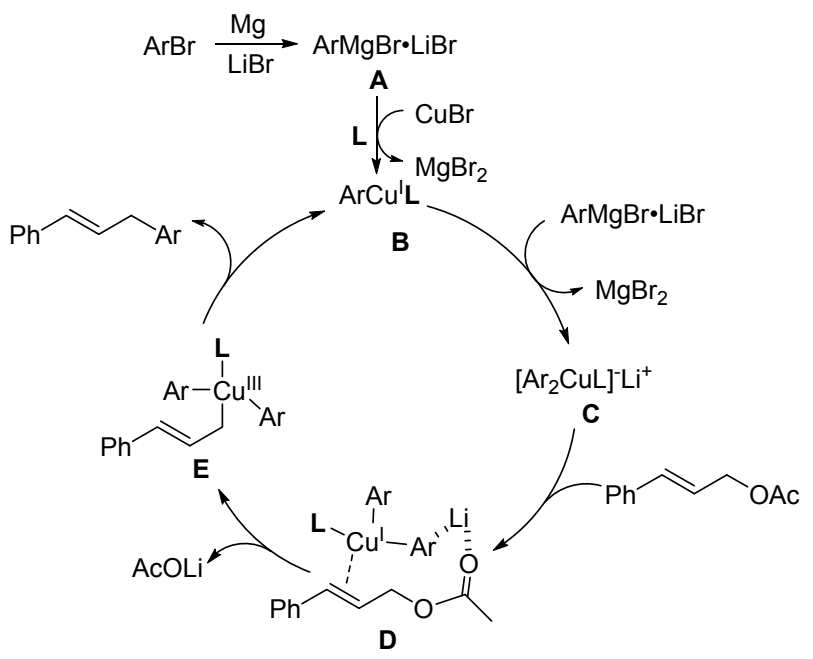

图式 2 可能的反应机理

Scheme 2 proposed mechanism

\section{2 结论}

本文通过一锅法策略实现了铜催化烯丙基乙酸酯 和卤代芳、烷烃的高选择性烯丙基化反应, 避免了常规 方法中预制格氏试剂的制备. 该方法所使用的原料价廉 易得, 方法简便, 底物适用性好, 具有较高收率及较好 的区域选择性.

\section{3 实验部分}

\section{1 仪器与试剂}

${ }^{1} \mathrm{H}$ NMR 和 ${ }^{13} \mathrm{C}$ NMR 使用 Bruker Avance III (500 $\mathrm{MHz}$ )核磁共振仪测定; 高分辨质谱使用 Bruker SolariX
70 FT-Ms 超高分辨四极杆串联傅立叶变换质谱仪测定. 实验所需试剂、溶剂除特殊说明外均为市售, 无需干燥 纯化处理.

\section{2 实验方法}

3.2 .1 铜催化烯丙基乙酸酯和卤代芳烃、烷烃的烯丙 基化反应

在 $10 \mathrm{~mL}$ 的反应管中依次加入烯丙基乙酸酯 $(0.5$ $\mathrm{mmol})$, 卤代烃 $(1.0 \mathrm{mmol})$, 溴化亚铜 $(0.05 \mathrm{mmol})$, 三苯 基膦(0.1 mmol), 镁屑(1.2 mmol)和 $1.5 \mathrm{~mL}$ 四氢呋喃, 然 后加入搅拌子, 经密封后于 $80{ }^{\circ} \mathrm{C}$ 油浴中反应 $6 \mathrm{~h}$. 待反 应体系冷却至室温后，向反应液中加入饱和氯化铵溶液 (3.0 mL)淬灭, 用乙酸乙酯 $(5.0 \mathrm{~mL} \times 3)$ 萃取. 有机相经 饱和氯化钠溶液 $(5.0 \mathrm{~mL})$ 洗涤, 无水硫酸钠干燥后减压 浓缩除去溶剂, 所得粗产品经柱层析(石油醚)分离后即 得相应的烯丙基类化合物.

1-肉桂基-4-甲基苯(3a) ${ }^{[15]}$ : 无色油状液体 $(89.6 \mathrm{mg}$, 产率 86\%). ${ }^{1} \mathrm{H}$ NMR $\left(500 \mathrm{MHz}, \mathrm{CDCl}_{3}\right) \delta: 7.33(\mathrm{~d}, J=7.5$ $\mathrm{Hz}, 2 \mathrm{H}), 7.26$ (t, $J=7.6 \mathrm{~Hz}, 2 \mathrm{H}), 7.17$ (t, $J=7.3 \mathrm{~Hz}, 1 \mathrm{H})$, $7.14 \sim 7.06(\mathrm{~m}, 4 \mathrm{H}), 6.42(\mathrm{~d}, J=15.8 \mathrm{~Hz}, 1 \mathrm{H}), 6.35 \sim 6.27$ $(\mathrm{m}, 1 \mathrm{H}), 3.48(\mathrm{~d}, J=6.7 \mathrm{~Hz}, 2 \mathrm{H}), 2.31(\mathrm{~s}, 3 \mathrm{H}) ;{ }^{13} \mathrm{C} \mathrm{NMR}$ $\left(125 \mathrm{MHz}, \mathrm{CDCl}_{3}\right) \delta: 137.4,137.0,135.6,130.7,129.4$, $129.1,128.5,128.4,127.0,126.0,38.9,21.0$.

1-肉桂基-2-甲基苯 $(3 \mathbf{b})^{[15]}$ : 无色油状液体 $(92.7 \mathrm{mg}$, 产率 89\%). ${ }^{1} \mathrm{H} \mathrm{NMR}\left(500 \mathrm{MHz}, \mathrm{CDCl}_{3}\right) \delta$ : $7.32(\mathrm{~d}, J=7.1$ $\mathrm{Hz}, 2 \mathrm{H}), 7.27$ (t, $J=7.6 \mathrm{~Hz}, 2 \mathrm{H}), 7.17$ (m, 5H), 6.40 6.27 $(\mathrm{m}, 2 \mathrm{H}), 3.51(\mathrm{~d}, J=5.0 \mathrm{~Hz}, 2 \mathrm{H}), 2.32(\mathrm{~s}, 3 \mathrm{H}) ;{ }^{13} \mathrm{C} \mathrm{NMR}$ $\left(125 \mathrm{MHz}, \mathrm{CDCl}_{3}\right) \delta: 138.1,137.5,136.4,130.8,130.2$, 129.2, 128.5, 128.4, 127.0, 126.4, 126.1, 126.0, 36.8, 19.4.

2-肉桂基-1,3-二甲苯 $(3 \mathbf{c})^{[16]}$ : 无色油状液体 $(100$ $\mathrm{mg}$, 产率 90\%). ${ }^{1} \mathrm{H}$ NMR $\left(500 \mathrm{MHz}, \mathrm{CDCl}_{3}\right) \delta: 7.31 \sim$ $7.20(\mathrm{~m}, 4 \mathrm{H}), 7.15$ (t, $J=7.2 \mathrm{~Hz}, 1 \mathrm{H}), 7.04$ (d, $J=2.9 \mathrm{~Hz}$, $3 \mathrm{H}), 6.31 \sim 6.17(\mathrm{~m}, 2 \mathrm{H}), 3.54(\mathrm{~d}, J=5.3 \mathrm{~Hz}, 2 \mathrm{H}), 2.33(\mathrm{~s}$, $6 \mathrm{H}) ;{ }^{13} \mathrm{C}$ NMR $\left(125 \mathrm{MHz}, \mathrm{CDCl}_{3}\right) \delta: 137.5,136.7,136.1$, 129.9, 128.4, 128.1, 127.3, 126.9, 126.1, 126.0, 32.9, 20.0.

1-肉桂基-4-氟苯 $(3 \mathrm{~d}){ }^{[15]}$ : 无色油状液体 $(88.1 \mathrm{mg}$, 产率 $83 \%$ ). ${ }^{1} \mathrm{H} \mathrm{NMR}\left(500 \mathrm{MHz}, \mathrm{CDCl}_{3}\right) \delta: 7.34(\mathrm{~d}, J=7.7$ $\mathrm{Hz}, 2 \mathrm{H}), 7.28$ (t, $J=7.6 \mathrm{~Hz}, 2 \mathrm{H}), 7.22 \sim 7.14(\mathrm{~m}, 3 \mathrm{H}), 6.98$ (t, $J=8.6 \mathrm{~Hz}, 2 \mathrm{H}), 6.42(\mathrm{~d}, J=15.8 \mathrm{~Hz}, 1 \mathrm{H}), 6.34 \sim 6.28$ $(\mathrm{m}, 1 \mathrm{H}), 3.50(\mathrm{~d}, J=6.7 \mathrm{~Hz}, 2 \mathrm{H}) ;{ }^{13} \mathrm{C} \mathrm{NMR}(125 \mathrm{MHz}$, $\left.\mathrm{CDCl}_{3}\right) \delta: 161.4(\mathrm{~d}, J=243.8 \mathrm{~Hz}), 137.3,135.7$ (d, $J=3.1$ $\mathrm{Hz}), 131.2,130.0$ (d, $J=7.8 \mathrm{~Hz}), 128.9,128.5,127.2$, 126.1, $115.2(\mathrm{~d}, J=21.1 \mathrm{~Hz}), 38.5$.

1 -氯-4-肉桂基苯 $(3 \mathrm{e})^{[15]}$ : 无色油状液体 $(100.6 \mathrm{mg}$, 产率 88\%). ${ }^{1} \mathrm{H}$ NMR (500 MHz, $\left.\mathrm{CDCl}_{3}\right) \delta: 7.34(\mathrm{~d}, J=7.7$ $\mathrm{Hz}, 2 \mathrm{H}), 7.28(\mathrm{dd}, J=15.8,7.9 \mathrm{~Hz}, 4 \mathrm{H}), 7.23 \sim 7.18(\mathrm{~m}$, 
1H), $7.15(\mathrm{~d}, J=8.1 \mathrm{~Hz}, 2 \mathrm{H}), 6.43(\mathrm{~d}, J=15.8 \mathrm{~Hz}, 1 \mathrm{H})$, $6.33 \sim 6.27(\mathrm{~m}, 1 \mathrm{H}), 3.49(\mathrm{~d}, J=6.8 \mathrm{~Hz}, 2 \mathrm{H}) ;{ }^{13} \mathrm{C} \mathrm{NMR}$ (125 MHz, $\left.\mathrm{CDCl}_{3}\right) \delta: 138.5,137.2,131.9,131.4,130.0$, 128.5, 128.5, 128.5, 127.2, 126.1, 38.6.

1-肉桂基-4-(三氟甲基)苯 (3f $)^{[15]}$ : 无色油状液体 (119.3 mg, 产率 91\%). ${ }^{1} \mathrm{H}$ NMR $\left(500 \mathrm{MHz}, \mathrm{CDCl}_{3}\right) \delta$ : $7.53(\mathrm{~d}, \quad J=7.9 \mathrm{~Hz}, 2 \mathrm{H}), 7.41 \sim 7.23(\mathrm{~m}, 6 \mathrm{H}), 7.20(\mathrm{t}, J=$ $7.3 \mathrm{~Hz}, 1 \mathrm{H}), 6.44(\mathrm{~d}, J=15.8 \mathrm{~Hz}, 1 \mathrm{H}), 6.32 \sim 6.26(\mathrm{~m}$, $1 \mathrm{H}), 3.55(\mathrm{~d}, J=6.9 \mathrm{~Hz}, 2 \mathrm{H}) ;{ }^{13} \mathrm{C} \mathrm{NMR}\left(125 \mathrm{MHz}, \mathrm{CDCl}_{3}\right)$ $\delta: 144.3,137.1,131.9,128.9,128.56(\mathrm{q}, J=10.3 \mathrm{~Hz})$, $128.5,127.8,127.4,126.1,125.4$ (q, $J=3.9 \mathrm{~Hz}), 123.3$, 39.0 .

1-肉桂基-4-甲氧基苯(3g) ${ }^{[15]}$ : 无色油状液体(89.7 $\mathrm{mg}$, 产率 80\%). ${ }^{1} \mathrm{H} \mathrm{NMR}\left(500 \mathrm{MHz}, \mathrm{CDCl}_{3}\right) \delta: 7.35$ (d, $J=7.7 \mathrm{~Hz}, 2 \mathrm{H}), 7.28(\mathrm{t}, J=7.6 \mathrm{~Hz}, 2 \mathrm{H}), 7.21 \sim 7.12(\mathrm{~m}$, $3 \mathrm{H}), 6.90 \sim 6.78(\mathrm{~m}, 2 \mathrm{H}), 6.42(\mathrm{~d}, J=15.8 \mathrm{~Hz}, 1 \mathrm{H}), 6.36 \sim$ $6.30(\mathrm{~m}, 1 \mathrm{H}), 3.79(\mathrm{~s}, 3 \mathrm{H}), 3.48(\mathrm{~d}, J=6.7 \mathrm{~Hz}, 2 \mathrm{H}) ;{ }^{13} \mathrm{C}$ NMR $\left(125 \mathrm{MHz}, \mathrm{CDCl}_{3}\right) \delta: 158.0,137.4,132.1,130.7$, 129.6, 129.6, 128.5, 127.0, 126.0, 113.8, 55.2, 38.4.

4-肉桂基- $N, N$-二甲基苯胺 $(3 \mathbf{h})^{[15]}$ : 棕色油状液体 (93.7 mg, 产率 79\%). ${ }^{1} \mathrm{H}$ NMR (500 MHz, $\mathrm{CDCl}_{3}$ ) $\delta: 7.32$ (d, $J=7.7 \mathrm{~Hz}, 2 \mathrm{H}), 7.25(\mathrm{t}, J=7.5 \mathrm{~Hz}, 2 \mathrm{H}), 7.15(\mathrm{t}, J=$ $7.3 \mathrm{~Hz}, 1 \mathrm{H}), 7.10(\mathrm{~d}, J=8.3 \mathrm{~Hz}, 2 \mathrm{H}), 6.70(\mathrm{~d}, J=8.3 \mathrm{~Hz}$, $2 \mathrm{H}), 6.40(\mathrm{~d}, J=15.8 \mathrm{~Hz}, 1 \mathrm{H}), 6.35 \sim 6.30(\mathrm{~m}, 1 \mathrm{H}), 3.43$ (d, $J=6.6 \mathrm{~Hz}, 2 \mathrm{H}), 2.87$ (s, 6H); ${ }^{13} \mathrm{C}$ NMR $(125 \mathrm{MHz}$, $\left.\mathrm{CDCl}_{3}\right) \delta: 149.1,137.5,130.2,130.1,129.2,128.3,128.1$, 126.8, 126.0, $113.040 .8,38.3$.

5-肉桂基苯并 $[\mathrm{d}][1,3]$ 二恶唑 $(3 \mathbf{3 i})^{[15]}$ : 无色油状液体 (100.1 mg, 产率 84\%). ${ }^{1} \mathrm{H}$ NMR $\left(500 \mathrm{MHz}, \mathrm{CDCl}_{3}\right) \delta$ : 7.35 (d, $J=7.2 \mathrm{~Hz}, 2 \mathrm{H}), 7.28$ (t, $J=7.6 \mathrm{~Hz}, 2 \mathrm{H}), 7.19$ (t, $J=7.3 \mathrm{~Hz}, 1 \mathrm{H}), 6.78 \sim 6.71(\mathrm{~m}, 2 \mathrm{H}), 6.68(\mathrm{~d}, J=7.9 \mathrm{~Hz}$, $1 \mathrm{H}), 6.42(\mathrm{~d}, J=15.8 \mathrm{~Hz}, 1 \mathrm{H}), 6.33 \sim 6.27(\mathrm{~m}, 1 \mathrm{H}), 5.91$ (s, 2H), $3.45(\mathrm{~d}, J=6.8 \mathrm{~Hz}, 2 \mathrm{H}) ;{ }^{13} \mathrm{C}$ NMR $(125 \mathrm{MHz}$, $\left.\mathrm{CDCl}_{3}\right) \delta: 147.6,137.3,133.9,130.9,129.3,128.5,128.4$, 127.1, 126.1, 121.3, 109.1, 108.2, 100.8, 39.0.

1-肉桂基菜 $(\mathbf{3 j})^{[15]}$ : 无色油状液体 $(103.8 \mathrm{mg}$, 产率 85\%). ${ }^{1} \mathrm{H}$ NMR (500 MHz, $\left.\mathrm{CDCl}_{3}\right) \delta: 8.07(\mathrm{~d}, J=8.1 \mathrm{~Hz}$, 1H), 7.85 (dd, $J=7.6,1.9 \mathrm{~Hz}, 1 \mathrm{H}), 7.74(\mathrm{~d}, J=8.0 \mathrm{~Hz}$, $1 \mathrm{H}), 7.51 \sim 7.45(\mathrm{~m}, 2 \mathrm{H}), 7.43 \sim 7.35(\mathrm{~m}, 2 \mathrm{H}), 7.31(\mathrm{~d}, J=$ $7.3 \mathrm{~Hz}, 2 \mathrm{H}), 7.25(\mathrm{t}, J=7.6 \mathrm{~Hz}, 2 \mathrm{H}), 7.20 \sim 7.14(\mathrm{~m}, 1 \mathrm{H})$, $6.54 \sim 6.41(\mathrm{~m}, 2 \mathrm{H}), 3.98(\mathrm{~d}, J=5.1 \mathrm{~Hz}, 2 \mathrm{H}) ;{ }^{13} \mathrm{C}$ NMR $\left(125 \mathrm{MHz}, \mathrm{CDCl}_{3}\right) \delta: 137.4,136.2,133.8,131.9,131.2$, $128.8,128.7,128.4,127.1,127.0,126.4,126.1,125.9$, 125.6, 125.56, 124.0, 36.4 .

2-肉桂基呋喃(3k $)^{[16]}$ : 无色油状液体(70.0 mg, 产率
76\%). ${ }^{1} \mathrm{H}$ NMR (500 MHz, $\left.\mathrm{CDCl}_{3}\right) \delta: 7.36 \sim 7.29(\mathrm{~m}, 3 \mathrm{H})$, $7.26(\mathrm{t}, J=7.6 \mathrm{~Hz}, 2 \mathrm{H}), 7.17(\mathrm{t}, J=7.3 \mathrm{~Hz}, 1 \mathrm{H}), 6.45(\mathrm{~d}$, $J=15.8 \mathrm{~Hz}, 1 \mathrm{H}), 6.30 \sim 6.25(\mathrm{~m}, 2 \mathrm{H}), 6.09 \sim 5.97(\mathrm{~m}, 1 \mathrm{H})$, $3.51(\mathrm{~d}, J=6.8 \mathrm{~Hz}, 2 \mathrm{H}) ;{ }^{13} \mathrm{C} \mathrm{NMR}\left(125 \mathrm{MHz}, \mathrm{CDCl}_{3}\right) \delta$ : $153.8,141.2,137.1,131.8,128.4,127.2,126.1,125.4$, $110.3,105.6,31.7$.

2-肉桂基噻吩(3I) ${ }^{[16]}$ : 无色油状液体(41.1 mg, 产率 41\%); ${ }^{1} \mathrm{H}$ NMR (500 MHz, $\left.\mathrm{CDCl}_{3}\right) \delta: 7.37(\mathrm{~d}, J=7.7 \mathrm{~Hz}$, 2H), 7.30 (t, $J=7.6 \mathrm{~Hz}, 2 \mathrm{H}), 7.26 \sim 7.19(\mathrm{~m}, 1 \mathrm{H}), 7.16$ (d, $J=5.1 \mathrm{~Hz}, 1 \mathrm{H}), 6.96 \sim 9.94(\mathrm{~m}, 1 \mathrm{H}), 6.86(\mathrm{~d}, J=3.3 \mathrm{~Hz}$, $1 \mathrm{H}), 6.51(\mathrm{~d}, J=15.8 \mathrm{~Hz}, 1 \mathrm{H}), 6.37$ (dt, $J=15.6,6.9 \mathrm{~Hz}$, $1 \mathrm{H}), 3.74(\mathrm{~d}, J=6.8 \mathrm{~Hz}, 2 \mathrm{H}) ;{ }^{13} \mathrm{C} \mathrm{NMR}\left(125 \mathrm{MHz}, \mathrm{CDCl}_{3}\right)$ $\delta: 143.1,137.1,131.3,128.5,128.1,127.3,126.9,126.2$, 124.6, 123.7, 33.3.

(E)-1-丁烯-1,4-二甲苯基二苯 $(3 \mathbf{m})^{[17]}$ : 无色油状液 体(65.6 mg, 产率 63\%). ${ }^{1} \mathrm{H}$ NMR $\left(500 \mathrm{MHz}, \mathrm{CDCl}_{3}\right) \delta$ : $7.34 \sim 7.26(\mathrm{~m}, 6 \mathrm{H}), 7.23 \sim 7.18(\mathrm{~m}, 4 \mathrm{H}), 6.41(\mathrm{~d}, J=15.7$ $\mathrm{Hz}, 1 \mathrm{H}), 6.29 \sim 6.23(\mathrm{~m}, 1 \mathrm{H}), 2.79(\mathrm{t}, J=7.8 \mathrm{~Hz}, 2 \mathrm{H}), 2.53$ (q, $J=7.5 \mathrm{~Hz}, 2 \mathrm{H}) ;{ }^{13} \mathrm{C}$ NMR $\left(125 \mathrm{MHz}, \mathrm{CDCl}_{3}\right) \delta: 141.7$, $137.6,130.3,129.9,128.5,128.4,128.3,126.9,125.9$, $125.9,35.8,34.9$.

(E)-己-1-烯-1,6-二基二苯 $(\mathbf{3 n})^{[18]}$ : 无色油状液体 (44.9 mg, 产率 38\%). ${ }^{1} \mathrm{H}$ NMR (500 MHz, $\mathrm{CDCl}_{3}$ ) $\delta: 7.33$ (d, $J=7.5 \mathrm{~Hz}, 2 \mathrm{H}), 7.30 \sim 7.26(\mathrm{~m}, 4 \mathrm{H}), 7.19(\mathrm{~d}, J=7.1$ $\mathrm{Hz}, 4 \mathrm{H}), 6.37(\mathrm{~d}, J=15.8 \mathrm{~Hz}, 1 \mathrm{H}), 6.24 \sim 6.18(\mathrm{~m}, 1 \mathrm{H})$, $2.64(\mathrm{t}, J=7.8 \mathrm{~Hz}, 2 \mathrm{H}), 2.24(\mathrm{q}, J=7.3 \mathrm{~Hz}, 2 \mathrm{H}), 1.71 \sim$ $1.65(\mathrm{~m}, 2 \mathrm{H}), 1.56-1.49(\mathrm{~m}, 2 \mathrm{H}) ;{ }^{13} \mathrm{C}$ NMR $(125 \mathrm{MHz}$, $\left.\mathrm{CDCl}_{3}\right) \delta: 142.6,137.8,130.8,129.9,128.5,128.4,128.3$, $126.8,125.9,125.7,35.9,32.9,31.1,29.0$.

(E) 庚-1-烯-1-苯 $(30)^{[19]}$ : 无色油状液体 $(16.6 \mathrm{mg}$, 产 率 19\%). ${ }^{1} \mathrm{H}$ NMR (500 MHz, $\left.\mathrm{CDCl}_{3}\right) \delta: 7.33$ (d, $J=7.3$ $\mathrm{Hz}, 2 \mathrm{H}), 7.28(\mathrm{t}, J=7.7 \mathrm{~Hz}, 2 \mathrm{H}), 7.18(\mathrm{t}, J=7.3 \mathrm{~Hz}, 1 \mathrm{H})$, $6.37(\mathrm{~d}, J=15.8 \mathrm{~Hz}, 1 \mathrm{H}), 6.25 \sim 6.19(\mathrm{~m}, 1 \mathrm{H}), 2.22 \sim 2.17$ (m, $2 \mathrm{H}), 1.50 \sim 1.44(\mathrm{~m}, 2 \mathrm{H}), 1.36 \sim 1.29(\mathrm{~m}, 4 \mathrm{H}), 0.90(\mathrm{t}$, $J=7.0 \mathrm{~Hz}, 3 \mathrm{H}) ;{ }^{13} \mathrm{C}$ NMR $\left(125 \mathrm{MHz}, \mathrm{CDCl}_{3}\right) \delta: 137.9$, $131.2,129.7,128.4,126.7,125.9,33.0,31.4,29.1,22.6$, 14.1 .

(E)-1- 甲氧基-4-(3-(对甲苯基)丙-1-烯-1-基)苯 (3aa) ${ }^{[20]}$ : 无色油状液体(98 mg, 产率 $83 \%$ ). ${ }^{1} \mathrm{H}$ NMR $\left(400 \mathrm{MHz}, \mathrm{CDCl}_{3}\right) \delta: 7.37 \sim 7.22(\mathrm{~m}, 3 \mathrm{H}), 7.16 \sim 7.08(\mathrm{~m}$, $3 \mathrm{H}), 6.83 \sim 6.81(\mathrm{~m}, 2 \mathrm{H}), 6.38(\mathrm{~d}, J=15.7 \mathrm{~Hz}, 1 \mathrm{H}), 6.23 \sim$ $6.16(\mathrm{~m}, 1 \mathrm{H}), 3.79$ (s, 3H), 3.48 (d, $J=6.8 \mathrm{~Hz}, 2 \mathrm{H}), 2.32$ (s, 3H); ${ }^{13} \mathrm{C}$ NMR $\left(100 \mathrm{MHz}, \mathrm{CDCl}_{3}\right) \delta$ : 158.8, 137.3, 135.54, 130.3, 130.2, 129.1, 128.5, 127.3, 127.2, 113.9, $55.2,38.9,21.0$. 
(E)-1- 甲基-4-(3-(4-(三氟甲基)苯基)烯丙基)苯

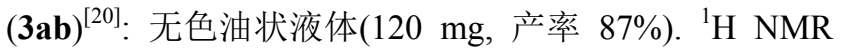
$\left(400 \mathrm{MHz}, \mathrm{CDCl}_{3}\right) \delta: 7.51(\mathrm{~d}, J=8.1 \mathrm{~Hz}, 2 \mathrm{H}), 7.47$ (d, $J=$ $8.1 \mathrm{~Hz}, 1 \mathrm{H}), 7.40$ (d, $J=8.1 \mathrm{~Hz}, 2 \mathrm{H}), 7.12(\mathrm{~s}, 3 \mathrm{H}), 6.43$ (s, $2 \mathrm{H}), 3.51(\mathrm{~d}, J=3.1 \mathrm{~Hz}, 2 \mathrm{H}), 2.32(\mathrm{~s}, 3 \mathrm{H}) ;{ }^{13} \mathrm{C}$ NMR $(100$ $\left.\mathrm{MHz}, \mathrm{CDCl}_{3}\right) \delta: 141.0,136.4,135.9,132.4,129.5,129.4$, $129.2,128.5,126.7,126.2,125.4(\mathrm{q}, J=4.2 \mathrm{~Hz}), 38.9$, 20.9.

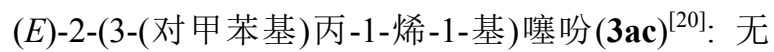
色油状液体(91.0 mg, 产率 85\%). ${ }^{1} \mathrm{H}$ NMR (400 MHz, $\left.\mathrm{CDCl}_{3}\right) \delta: 7.17 \sim 7.01(\mathrm{~m}, 5 \mathrm{H}), 6.95 \sim 6.80(\mathrm{~m}, 2 \mathrm{H}), 6.51$ (d, $J=15.6 \mathrm{~Hz}, 1 \mathrm{H}), 6.21 \sim 6.14(\mathrm{~m}, 1 \mathrm{H}), 3.44(\mathrm{~d}, J=6.8$ $\mathrm{Hz}, 2 \mathrm{H}), 2.31$ (s, 3H); ${ }^{13} \mathrm{C}$ NMR $\left(100 \mathrm{MHz}, \mathrm{CDCl}_{3}\right) \delta$ : $142.7,136.7,135.6,129.4,129.2,128.6,127.2,124.6$, 124.0, 123.4, 38.6, 21.0 .

(E)-1-甲基-4-(2-甲基-3-苯基烯丙基)苯(3ad) ${ }^{[21]}$ : 无 色油状液体(91.1 mg, 产率 82\%). ${ }^{1} \mathrm{H}$ NMR (400 MHz, $\left.\mathrm{CDCl}_{3}\right) \delta: 7.31 \sim 7.22(\mathrm{~m}, 4 \mathrm{H}), 7.19 \sim 7.08(\mathrm{~m}, 5 \mathrm{H}), 6.35$ (s, 1H), 3.42 (s, 2H), 2.31 (s, 3H), 1.78 (s, 3H); ${ }^{13} \mathrm{C}$ NMR $\left(100 \mathrm{MHz}, \mathrm{CDCl}_{3}\right) \delta: 138.4,138.3,136.7,135.6,129.0$, $128.8,128.0,126.5,126.0,46.7,21.0,17.6$.

1-烯丙基-4-甲基苯 (3ae) ${ }^{[20]}$ : 无色油状液体 (37.0 $\mathrm{mg}$, 产率 56\%). ${ }^{1} \mathrm{H}$ NMR (500 MHz, $\left.\mathrm{CDCl}_{3}\right) \delta: 7.09$ (q, $J=7.9 \mathrm{~Hz}, 4 \mathrm{H}), 5.99 \sim 5.91(\mathrm{~m}, 1 \mathrm{H}), 5.17 \sim 4.95(\mathrm{~m}, 2 \mathrm{H})$, 3.35 (d, $J=6.7 \mathrm{~Hz}, 2 \mathrm{H}), 2.32$ (s, 3H); ${ }^{13} \mathrm{C}$ NMR (125 MHz, $\left.\mathrm{CDCl}_{3}\right) \delta: 137.7,136.9,135.5,129.1,128.4,115.5,39.8$, 21.0 .

1-甲基-4-(3-甲基丁-2-烯-1-基)苯(3af) ${ }^{[22]}$ : 无色油状 液体(63.3 mg, 产率 79\%). ${ }^{1} \mathrm{H}$ NMR (500 MHz, $\left.\mathrm{CDCl}_{3}\right) \delta$ : $7.06(\mathrm{~d}, J=2.2 \mathrm{~Hz}, 4 \mathrm{H}), 5.38 \sim 5.24(\mathrm{~m}, 1 \mathrm{H}), 3.29$ (d, $J=$ $7.5 \mathrm{~Hz}, 2 \mathrm{H}), 2.30$ (s, 3H), 1.72 (d, $J=11.7 \mathrm{~Hz}, 6 \mathrm{H}) ;{ }^{13} \mathrm{C}$ NMR $\left(125 \mathrm{MHz}, \mathrm{CDCl}_{3}\right) \delta$ : 138.7 135.1, 132.1 129.0, 128.1, 123.5, 33.9, 25.7, 21.0, 17.8.

(E)-1-(3,7-二甲基辛基-2,6-二烯-1-基)-4-甲基苯 (3ag) ${ }^{[23]}$ : 无色油状液体(92.5 mg, 产率 81\%). ${ }^{1} \mathrm{H}$ NMR $\left(500 \mathrm{MHz}, \mathrm{CDCl}_{3}\right) \delta: 7.13 \sim 7.04(\mathrm{~m}, 4 \mathrm{H}), 5.37 \sim 5.28(\mathrm{~m}$, $1 \mathrm{H}), 5.12 \sim 5.09(\mathrm{~m}, 1 \mathrm{H}), 3.32(\mathrm{~d}, J=7.4 \mathrm{~Hz}, 2 \mathrm{H}), 2.31(\mathrm{~s}$, $3 \mathrm{H}), 2.10(\mathrm{t}, J=7.5 \mathrm{~Hz}, 2 \mathrm{H}), 2.05(\mathrm{~d}, J=7.7 \mathrm{~Hz}, 2 \mathrm{H}), 1.69$ (d, $J=8.5 \mathrm{~Hz}, 6 \mathrm{H}), 1.60$ (s, 3H); ${ }^{13} \mathrm{C}$ NMR $(125 \mathrm{MHz}$, $\left.\mathrm{CDCl}_{3}\right) \delta: 138.8,136.1,135.2,131.6,129.1,128.3,124.4$, 123.4, 39.9, 33.9, 26.7, 25.9, 21.1, 17.9, 16.2.

(E)-1-甲基-4-(4-苯基丁-3-烯-2-基)苯(3ah) ${ }^{[7 b]}$ : 无色 油状液体 $103.4 \mathrm{mg}$, 产率 93\%). ${ }^{1} \mathrm{H}$ NMR $(500 \mathrm{MHz}$, $\left.\mathrm{CDCl}_{3}\right) \delta: 7.33(\mathrm{~d}, J=7.4 \mathrm{~Hz}, 2 \mathrm{H}), 7.26(\mathrm{t}, J=7.1 \mathrm{~Hz}, 2 \mathrm{H})$, $7.19 \sim 7.08(\mathrm{~m}, 5 \mathrm{H}), 6.37(\mathrm{~d}, J=5.3 \mathrm{~Hz}, 2 \mathrm{H}), 3.65 \sim 3.52$ (m, 1H), 2.31 (s, 3H), 1.43 (d, $J=6.8 \mathrm{~Hz}, 3 \mathrm{H}) ;{ }^{13} \mathrm{C}$ NMR $\left(125 \mathrm{MHz}, \mathrm{CDCl}_{3}\right) \delta: 142.6,137.6,135.7,135.4,129.1$, 128.4, 128.3, 127.1, 126.9, 126.1, 42.1, 21.2, 21.0 .

(E)-1-甲基-4-(1-苯基戊-1-烯-3-基)苯(3ai) ${ }^{[24]}$ ：无色 油状液体(105.2 mg, 产率 89\%). ${ }^{1} \mathrm{H}$ NMR $(500 \mathrm{MHz}$, $\left.\mathrm{CDCl}_{3}\right) \delta: 7.33(\mathrm{~d}, J=7.7 \mathrm{~Hz}, 2 \mathrm{H}), 7.26(\mathrm{t}, J=7.6 \mathrm{~Hz}, 2 \mathrm{H})$, $7.15(\mathrm{~d}, J=21.6 \mathrm{~Hz}, 5 \mathrm{H}), 6.38(\mathrm{~d}, J=15.9 \mathrm{~Hz}, 1 \mathrm{H}), 6.31$ (dd, $J=15.8,7.5 \mathrm{~Hz}, 1 \mathrm{H}), 3.27$ (q, $J=7.4 \mathrm{~Hz}, 1 \mathrm{H}), 2.32$ (s, $3 \mathrm{H}), 1.83 \sim 1.77(\mathrm{~m}, 2 \mathrm{H}), 0.90(\mathrm{t}, J=7.4 \mathrm{~Hz}, 3 \mathrm{H}) ;{ }^{13} \mathrm{C}$ NMR $\left(125 \mathrm{MHz}, \mathrm{CDCl}_{3}\right) \delta: 141.5,137.7,135.6,134.4$, 129.2, 129.1, 128.4, 127.5, 126.9, 126.0, 50.5, 28.7, 21.0, 12.3 .

(E)-1-甲基-4-(5-甲基-1-苯基己-1-烯-3-基)苯(3aj): 无色油状液体(128.2 mg, 产率 97\%). ${ }^{1} \mathrm{H}$ NMR (500 $\left.\mathrm{MHz}, \mathrm{CDCl}_{3}\right) \delta: 7.33(\mathrm{~d}, J=7.5 \mathrm{~Hz}, 2 \mathrm{H}), 7.28 \sim 7.23(\mathrm{~m}$, $3 \mathrm{H}), 7.19 \sim 7.10(\mathrm{~m}, 5 \mathrm{H}), 6.37(\mathrm{~d}, J=15.9 \mathrm{~Hz}, 1 \mathrm{H}), 6.29$ (dd, $J=15.8,7.6 \mathrm{~Hz}, 1 \mathrm{H}), 3.48(\mathrm{q}, J=7.6 \mathrm{~Hz}, 1 \mathrm{H}), 2.32$ (s, 3H), 1.65 (t, $J=7.3 \mathrm{~Hz}, 2 \mathrm{H}), 1.54$ (d, $J=9.4 \mathrm{~Hz}, 2 \mathrm{H})$, 0.91 (s, 6H); ${ }^{13} \mathrm{C}$ NMR $\left(125 \mathrm{MHz}, \mathrm{CDCl}_{3}\right) \delta: 141.7,137.6$, 135.6, 134.8, 129.1, 128.8, 128.4, 127.4, 126.9, 126.1, 46.4, 45.1, 25.4, 22.6, 21.0. HRMS (ESI) calcd for $\mathrm{C}_{20} \mathrm{H}_{25}$ $[\mathrm{M}+\mathrm{H}]^{+}: 265.1951$, found 265.1948 .

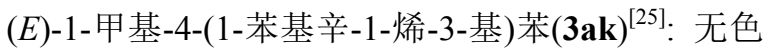
油状液体(112.8 mg, 产率 81\%). ${ }^{1} \mathrm{H}$ NMR $(500 \mathrm{MHz}$, $\left.\mathrm{CDCl}_{3}\right) \delta: 7.33(\mathrm{~d}, J=7.6 \mathrm{~Hz}, 2 \mathrm{H}), 7.29 \sim 7.20(\mathrm{~m}, 3 \mathrm{H})$, 7.17 (t, $J=7.3 \mathrm{~Hz}, 1 \mathrm{H}), 7.12$ (s, 4H), 6.37 (d, $J=15.9 \mathrm{~Hz}$, $1 \mathrm{H}), 6.31$ (dd, $J=15.8,7.4 \mathrm{~Hz}, 1 \mathrm{H}), 3.36(\mathrm{q}, J=7.4 \mathrm{~Hz}$, 1H), 2.32 (s, $4 \mathrm{H}), 1.77$ (q, $J=6.5 \mathrm{~Hz}, 2 \mathrm{H}), 1.33 \sim 1.21(\mathrm{~m}$, $6 \mathrm{H}), 0.86(\mathrm{~d}, J=6.2 \mathrm{~Hz}, 3 \mathrm{H}) ;{ }^{13} \mathrm{C}$ NMR $\left(125 \mathrm{MHz}, \mathrm{CDCl}_{3}\right)$ $\delta: 141.7,137.7,135.5,134.7,129.1,129.0,128.4,127.4$, $126.9,126.0,48.7,35.8,31.8,27.3,22.5,21.0,14.1$.

(E)-(3-(对甲苯基)丁-1-烯-1,4-二基)二苯(3al): 无色 油状液体(119.4 mg, 产率 80\%). ${ }^{1} \mathrm{H}$ NMR $(500 \mathrm{MHz}$, $\left.\mathrm{CDCl}_{3}\right) \delta: 7.24(\mathrm{dd}, J=15.3,7.5 \mathrm{~Hz}, 4 \mathrm{H}), 7.20 \sim 7.11(\mathrm{~m}$, $5 \mathrm{H}), 7.07(\mathrm{~d}, J=8.6 \mathrm{~Hz}, 6 \mathrm{H}), 6.37(\mathrm{dd}, J=15.9,7.4 \mathrm{~Hz}$, $1 \mathrm{H}), 6.25(\mathrm{~d}, J=15.9 \mathrm{~Hz}, 1 \mathrm{H}), 3.68(\mathrm{q}, J=7.5 \mathrm{~Hz}, 1 \mathrm{H})$, 3.08 (dd, $J=7.5,3.6 \mathrm{~Hz}, 2 \mathrm{H}), 2.29$ (s, 3H); ${ }^{13} \mathrm{C}$ NMR $(125$ $\left.\mathrm{MHz}, \mathrm{CDCl}_{3}\right) \delta: 140.7,140.0,137.5,135.7,133.4,129.7$, $129.2,129.1,128.3,128.0,127.7,127.0,126.1,125.8$, 50.1, 42.6, 21.0. HRMS (ESI) calcd for $\mathrm{C}_{23} \mathrm{H}_{23}: 299.1794$, found 299.1799 .

(E)-4,4'-(戊-1-烯-1,3-二基)双 (甲基苯)(3am) ${ }^{[24]}$ : 无 色油状液体(106.4 mg, 产率 85\%). ${ }^{1} \mathrm{HNMR}(500 \mathrm{MHz}$, $\left.\mathrm{CDCl}_{3}\right) \delta: 7.40(\mathrm{~d}, J=7.9 \mathrm{~Hz}, 2 \mathrm{H}), 7.33 \sim 7.26(\mathrm{~m}, 4 \mathrm{H})$, $7.24(\mathrm{~d}, \quad J=7.9 \mathrm{~Hz}, 2 \mathrm{H}), 6.53(\mathrm{~d}, J=15.9 \mathrm{~Hz}, 1 \mathrm{H}), 6.43$ 
(dd, $J=15.8,7.7 \mathrm{~Hz}, 1 \mathrm{H}), 3.42$ (q, $J=7.5 \mathrm{~Hz}, 1 \mathrm{H}), 2.49$ (s, $3 \mathrm{H}), 2.47(\mathrm{~s}, 3 \mathrm{H}), 1.99 \sim 1.94(\mathrm{~m}, 2 \mathrm{H}), 1.08(\mathrm{t}, J=7.4 \mathrm{~Hz}$, $3 \mathrm{H}) ;{ }^{13} \mathrm{C} \mathrm{NMR}\left(125 \mathrm{MHz}, \mathrm{CDCl}_{3}\right) \delta: 141.6,136.5,135.5$, $134.9,133.4,129.1,129.1,127.5,126.0,50.5,28.8,21.1$, 21.0, 12.3 .

(E)-1-氟-4-(3-(对甲苯基)戊-1-烯-1-基)苯(3an)：无 色油状液体(115.7 mg, 产率 91\%). ${ }^{1} \mathrm{H}$ NMR (500 MHz, $\left.\mathrm{CDCl}_{3}\right) \delta: 7.28(\mathrm{dd}, J=8.1,5.8 \mathrm{~Hz}, 2 \mathrm{H}), 7.12$ (s, 4H), 6.95 (t, $J=8.6 \mathrm{~Hz}, 2 \mathrm{H}), 6.33$ (d, $J=15.9 \mathrm{~Hz}, 1 \mathrm{H}), 6.22$ (dd, $J=$ 15.8, $7.8 \mathrm{~Hz}, 1 \mathrm{H}), 3.25$ (q, $J=7.4 \mathrm{~Hz}, 1 \mathrm{H}), 2.32$ (s, 3H), $1.82 \sim 1.76(\mathrm{~m}, 2 \mathrm{H}), 0.90(\mathrm{t}, J=7.3 \mathrm{~Hz}, 3 \mathrm{H}) ;{ }^{13} \mathrm{C}$ NMR $(12$ $\left.\mathrm{MHz}, \mathrm{CDCl}_{3}\right) \delta: 161.9(\mathrm{~d}, J=245.9 \mathrm{~Hz}), 141.3,135.7$, $134.2(\mathrm{~d}, J=2.1 \mathrm{~Hz}), 129.2,128.0,127.5,127.5,127.5$, $115.2(\mathrm{~d}, J=21.5 \mathrm{~Hz}), 50.5,28.7,21.0,12.3$. HRMS (ESI) calcd for $\mathrm{C}_{18} \mathrm{H}_{19} \mathrm{FNa}[\mathrm{M}+\mathrm{Na}]^{+}:$277.1363, found 277.1368.

辅助材料(Supporting Information) 所得化合物的 ${ }^{1} \mathrm{H}$ $\mathrm{NMR}$ 和 ${ }^{13} \mathrm{C} \mathrm{NMR}$ 图谱. 这些材料可以免费从本刊网站 (http://sioc-journal.cn/)上下载.

\section{References}

[1] (a) Zhang, Z.-Q.; Zhang, B.; Lu, X.; Liu, J.-H.; Lu, X.-Y.; Xiao, B.; Fu, Y. Org. Lett. 2016, 18, 952.

(b) Nguyen, T. N. T.; Thiel, N. O.; Pape, F.; Teichert, J. F. Org. Lett. 2016, 18, 2455.

(c) Xu, G.; Zhao, H.; Fu, B.; Cang, A.; Zhang, G.; Xiong, T.; Zhang, Q. Angew. Chem. Int.Ed. 2017, 56, 13130.

[2] (a) Trost, B. M.; Van Vranken, D. L. Chem. Rev. 1996, 96, 395.

(b) Falciola, C. A.; Alexakis, A. Eur. J. Org. Chem. 2008, 22, 3765.

(c) Cui, P.; Liu, H.; Zhang, D.; Wang, C. Chin. J. Org. Chem. 2012, 32, 1401 (in Chinese).

(崔朋雷, 刘海燕, 张冬暖, 王春, 有机化学, 2012, 32, 1401.) (d) Deng, Y.; Yang, W.; Yang, X.; Yang, D. Chin. J. Org. Chem. 2017, 37, 3039 (in Chinese)

(邓颖颖，杨文，杨新，杨定乔，有机化学，2017，37, 3039.)

(e) Zhang, H.; Gu, Q.; You, S. Chin. J. Org. Chem. 2019, 39, 15 (in Chinese).

(张慧君, 顾庆, 游书力, 有机化学, 2019, 39, 15.)

[3] (a) Han, X.; Zhang, Y.; Wu, J. J. Am. Chem. Soc. 2010, 132, 4104. (b) Lauer, A. M.; Mahmud, F.; Wu, J. J. Am. Chem. Soc. 2011, 133, 9119.

(c) Hornillos, V.; Pérez, M.; Fañanás-Mastral, M.; Feringa, B. L. J. Am. Chem. Soc. 2013, 135, 2140.

(d) Magrez, M.; Guen, Y. L.; Baslé, O.; Crévisy, C.; Mauduit, M.
Chem. Eur. J. 2013, 19, 1199.

[4] Liu, J.-H.; Yang, C.-T.; Lu, X.-Y.; Zhang, Z.-Q. Xu, L.; Cui, M.; Lu, X.; Xiao, B.; Fu, Y.; Liu, L. Chem. Eur. J. 2014, 20, 15334.

[5] (a) Li, Z.; Sun, H.-M.; Shen, Q. Org. Biomol. Chem. 2016, 14, 3314.

(b) Zhang, J.; Lu, G.; Xu, J.; Sun, H.; Shen, Q. Org. Lett. 2016, 18, 2860.

[6] (a) Czaplik, W. M.; Mayer, M.; Jacobi von Wangelin, A. Angew. Chem. Int. Ed. 2009, 48, 607.

(b) Mayer, M.; Czaplik, W. M.; Jacobi von Wangelin, A. Adv. Synth. Catal. 2010, 352, 2147.

[7] (a) Cui, X.; Wang, S.; Zhang, Y.; Deng, W.; Qian, Q.; Gong, H. Org. Biomol. Chem. 2013, 11, 3094.

(b) Chen, H.; Jia, X.; Yu, Y.; Gong, H. Angew. Chem. Int. Ed. 2017, 56, 13103 .

[8] Anka-Lufford, L. L.; Prinsell, M. R.; Weix, D. J. J. Org. Chem. 2012, 77, 9989.

[9] Qian, X.; Auffrant, A.; Felouat, A.; Gosmini, C. Angew. Chem. Int. Ed. 2011, 50, 10402

[10] Liu, Y.; Fang, Y.; Zhang, L.; Jing, X.; Li, R.; Zhu, S.; Gao, H.; Fang, J.; Xia, Q. Chin. J. Org. Chem. 2014, 34, 1523 (in Chinese). (刘雨燕, 方烨汶, 张莉, 金小平, 李瑞丰, 朱帅汝, 高浩其, 房 江华，夏勤波，有机化学, 2014, 34, 1523.)

[11] Tseng, C. C.; Paisley, S. D.; Goering, H. L. J. Org. Chem. 1986, 51 , 2884.

[12] Pérez, M.; Fañanás-Mastral, M.; Bos, P. H.; Rudolph, A.; Harutyunyan, S. R.; Feringa, B. L. Nat. Chem. 2011, 3, 377.

[13] (a) Persson, E. S. M.; Klaveren, M. V.; Grove, D. M.; Bäckvall, J. E.; Koten, G. V. Chem. Eur. J. 1995, 1, 351.

(b) Bertz, S. H.; Cope, S.; Murphy, M.; Ogle, C. A.; Taylor, B. J. J. Am. Chem. Soc. 2007, 129, 7208 .

[14] (a) Bartholomew, E. R.; Bertz, S. H.; Cope, S.; Murphy, M.; Ogle, C. A. J. Am. Chem. Soc. 2008, 130, 11244.

(b) Xu, H.; Man, Q.; Lin, Y.; Li, Y.; Feng, Y. Chin. J. Org. Chem. 2010, 30,9 (in Chinese).

(许华建，蔄秋石，林义成，李源源，冯乙已，有机化学， 2010, 30, 9.)

[15] Nguyen, T. N. T.; Thiel, N. O.; Teichert, J. F. Chem. Commun. 2017, 53, 11686 .

[16] Yang, B.; Wang, Z.-X. J. Org. Chem. 2017, 82, 4542

[17] Frlan, R.; Sova, M.; Gobec, S.; Stavber, G.; Časar, Z. J. Org. Chem. 2015, 80, 7803.

[18] Franco, T. D.; Epenoy, A.; Hu, X. Org. Lett. 2015, 17, 4910.

[19] Kim, H. J.; Su, L.; Jung, H.; Koo, S. Org. Lett. 2011, 13, 2682.

[20] Guo, S.; Yuan, Y.; Xiang, J. New J. Chem. 2015, 39, 3093.

[21] Zhu, Z.-F.; Tu,. J-L.; Liu, F. Chem. Commun. 2019, 55, 11478.

[22] Mayer, M.; Czaplik, W. M.; Jacobi von Wangelin, A. Adv. Synth. Catal. 2010, 352, 2147.

[23] Tamaru, Y.; Kagotani, M.; Yoshida, Z.-I. J. Chem. Soc., Chem. Commun. 1978, 8, 367.

[24] Deng, W.-H.; Ye, F.; Bai, X.-F.; Li, L.; Song, T.; Wei, Y.-L.; Xu, L.-W. $R S C A d v$. 2014, 4, 479

[25] Kobayashi, Y.; Tokoro, Y.; Watatani, K. Eur. J. Org. Chem. 2000, 23,3825 . 